\title{
LAMINAÇÃO \\ PREVISÃo DA EVOLUÇÃo TÉRMICA DURANTE A LAMINAÇÃO A QUENTE DOS AÇOS*
}

Antônio Adel dos Santos ${ }^{1}$ Cristóvão Nery Giacomin²

Bruno Alves Resende 3

\section{Resumo}

O controle da temperatura é fundamental no processo de laminação a quente dos aços, motivando a dedicação de esforços em estudos sobre a transferência de calor e na aplicação de melhorias operacionais. Neste sentido, o objetivo deste trabalho é apresentar o desenvolvimento, os resultados e as aplicações de modelos de cálculo da evolução de temperatura do aço durante a laminação a quente, desde o reaquecimento da placa até o produto laminado e resfriado na forma de chapas. São mostradas formulações matemáticas, métodos numéricos de solução usados, validação dos cálculos e a contribuição dos modelos para o entendimento e melhoria de processo. Todo esse desenvolvimento foi baseado em trabalhos realizados na Usiminas, nos últimos anos, cuja fundamentação teórica, buscada na literatura, foi adaptada e validada de acordo com as especificidades de cada linha. Além disso, os modelos foram implantados como simuladores offline, tornando prática sua aplicação pelos analistas de processo. São mostrados exemplos de cálculo do aquecimento de placas, da evolução térmica das placas nos fornos até a entrada dos laminadores, e da evolução do esboço durante a laminação de chapas grossas.

Palavras-chave: Laminação a quente; Evolução térmica; Modelo matemático.

\section{PREDICTION OF TEMPERATURE EVOLUTION DURING HOT ROLLING OF STEELS}

\section{Abstract}

Temperature control is of paramount importance during hot rolling of steel, thus motivating the application of efforts on both heat transfer investigations and improvement of operating practices. Therefore, the aim of this work is to present the development, results and application examples of modeling the temperature evolution of steel during hot rolling, from the slab reheating process up to the as rolled product after its cooling. Basic features are shown such as model formulations, numerical methods, validations and their contribution to the process understanding and improvement. All the work was based on recent development carried out at Usiminas. The theoretical background found in literature was adapted and fitted to each processing line particularity. In addition, the models were implemented as offline simulation tools, making easy their use for technical staff. Examples are given of the slab reheating process, thermal evolution of slabs from the furnace up to the first rougher and the temperature change during hot rolling of plates.

Keywords: Hot rolling; Temperature evolution; Mathematical models.

1 Membro da ABM; Engenheiro Metalurgista, D.Sc., CQE/ASQ, Centro de Tecnologia Usiminas, Ipatinga, $M G$.

2 Membro da ABM; Analista de TI, Centro de Tecnologia Usiminas; Ipatinga, MG.

3 Engenheiro Mecânico, MSc., Centro de Tecnologia da Usiminas; Ipatinga, MG.

* Contribuição técnica ao $51^{\circ}$ Seminário de Laminação - Processos e Produtos Laminados e Revestidos, 28 a 31 de outubro de 2014, Foz do Iguaçu, PR, Brasil. 


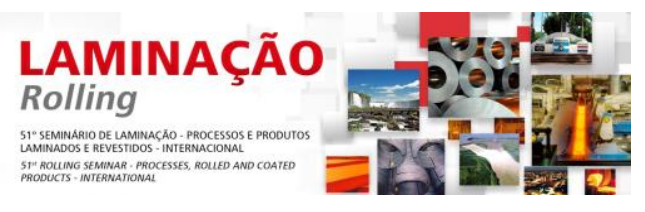

\section{INTRODUÇÃO}

O conhecimento e o controle da temperatura do material durante o processo de laminação a quente é fundamental em termos de produtividade, qualidade do produto e custos de produção. Para que todos estes requisitos sejam atendidos ao mesmo tempo, dentro de suas faixas de aceitação, aplicam-se, usualmente, modelos de previsão da evolução da temperatura no processo, aliados a medições realizadas ao longo das linhas de laminação. Os modelos matemáticos de temperatura são baseados nas leis fundamentais de transferência de calor entre o material e o meio ambiente, levando em consideração as características das linhas e dos materiais processados.

O trabalho pioneiro sobre modelos de evolução térmica em linhas de laminação a quente foi realizado por Hollander [1]. Hoje, encontra-se disponível vasta literatura técnica sobre transferência de calor neste processo, por exemplo, para as etapas de reaquecimento [2,3], resfriamento final do produto laminado [4-6] e laminação propriamente [7]. Ênfase tem sido dada à etapa de resfriamento final do laminado, especialmente na produção de aços de alta resistência, por este processo ser preponderante na definição das propriedades mecânicas finais dos aços laminados a quente.

No entanto, detalhes de aplicações dos modelos aos processos industriais são mais escassos na literatura. Ao menos em parte, isto pode ser atribuído ao fato que nas usinas siderúrgicas os modelos de controle online existentes são, normalmente, propriedade de empresas fornecedoras de tecnologias de processo, que se resguardam de publicações detalhadas.

Neste sentido, o objetivo deste trabalho é apresentar uma contribuição ao entendimento e à divulgação de aspectos básicos da aplicação de modelos matemáticos de transferência de calor ao processo de laminação a quente dos aços. São destacados alguns desenvolvimentos realizados na Usiminas, nos últimos anos, relativos a modelos de previsão da evolução de temperatura, desde o reaquecimento de placa até o resfriamento final do laminado. São mostrados exemplos de formulação matemática, solução numérica, forma de representação da realidade dos processos nos modelos e aspectos de sua validação e aplicação. Tais exemplos são relacionados às linhas de laminação de chapas grossas e de tiras a quente da Usiminas, em lpatinga

\section{DESENVOLVIMENTO DOS MODELOS MATEMÁTICOS}

Em todas as etapas da laminação a quente, a temperatura do material varia com o tempo, o que caracteriza um problema transiente de transferência de calor. Além disto, o material está em movimento nestas etapas, exceto no resfriamento da bobina a quente após estocagem. Então, além de transiente, o problema é dinâmico. Portanto, o primeiro aspecto fundamental para o desenvolvimento dos modelos é a determinação das relações de posição, tempo e velocidade do material. Cada posição do material precisa ser associada ao regime de resfriamento apropriado.

Em seguida, tem-se o desenvolvimento de soluções numéricas para o problema transiente de transferência de calor, com suas condições de contorno e inicial. A questão crucial do desenvolvimento é a determinação realística dos fluxos de calor entre o ambiente e o material, que são caracterizados pelos coeficientes de transferência de calor.

\footnotetext{
* Contribuição técnica ao $51^{\circ}$ Seminário de Laminação - Processos e Produtos Laminados e Revestidos, 28 a 31 de outubro de 2014, Foz do Iguaçu, PR, Brasil.
} 


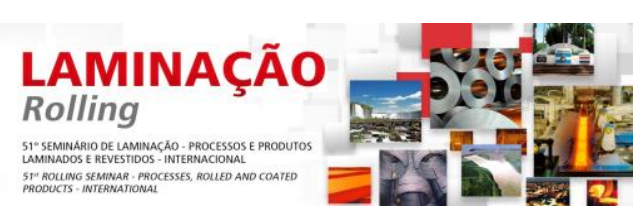

\subsection{Diagramas TPV - Tempo-Posição-Velocidade}

No levantamento dos diagramas TPV são consideradas: (a) configuração geométrica da linha ou processo, com distâncias relevantes; (b) forma de deslocamento do material, incluindo aceleração, desaceleração, movimento uniforme e paradas; (c) simplificações assumidas que são necessárias para viabilizar e/ou facilitar o desenvolvimento dos algoritmos. O diagrama TPV pode ser uma característica intrínseca da configuração e modo de operação da linha ou equipamento, ou estar acoplado aos modelos de evolução térmica.

No caso do modelo de reaquecimento desenvolvido [2], considerou-se o deslocamento da placa com velocidade constante no forno, ou, ao menos, constante em cada zona, podendo ser diferente entre elas. Dado o comprimento do forno, ou de cada zona, e o respectivo tempo de permanência, a determinação da velocidade é imediata. O gráfico posição versus tempo da placa será linear com inclinação positiva.

Em seguida, a placa é desenfornada e segue até o primeiro laminador. Na figura 1 é apresentado o diagrama de velocidade da placa em função da posição para a Linha de Tiras a Quente, desde os fornos de reaquecimento até a entrada do primeiro laminador, R1. Este diagrama foi construído para o simulador de evolução térmica da placa.

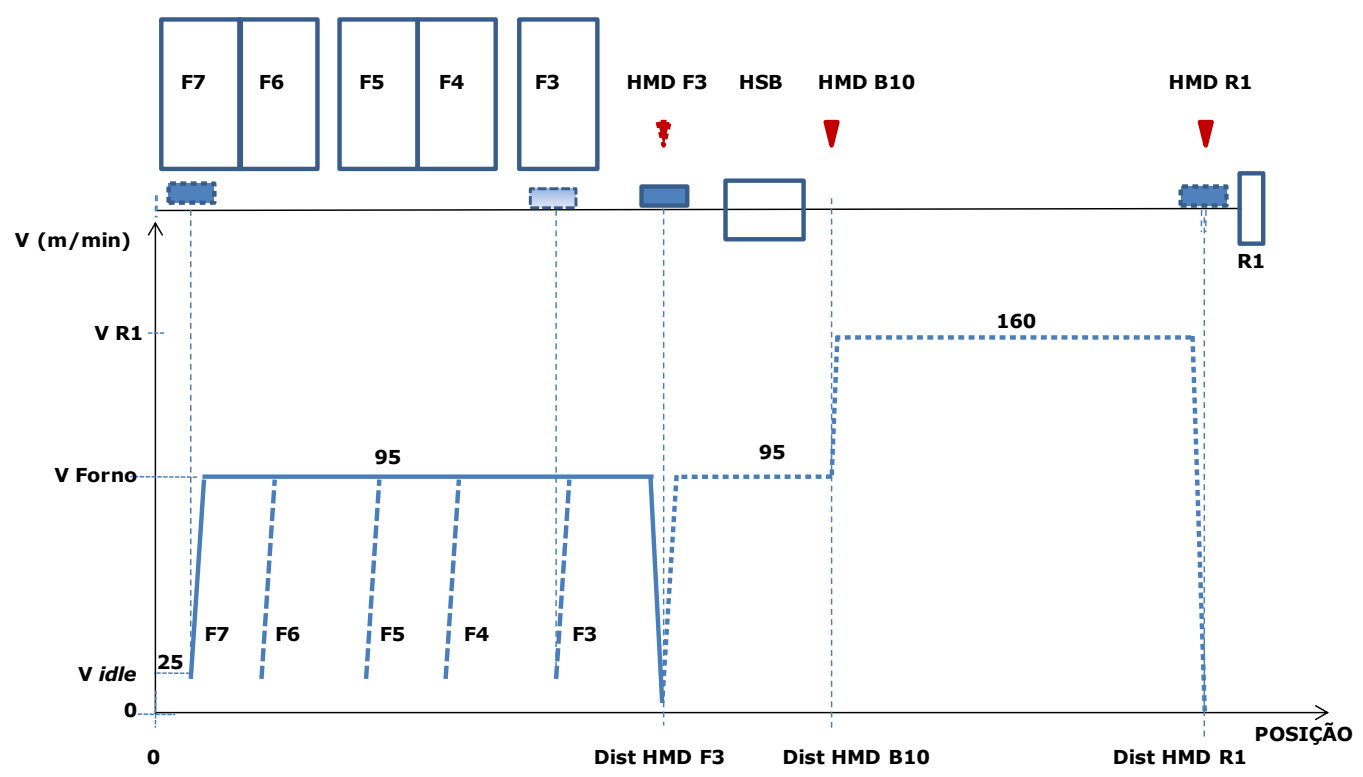

Figura 1. Diagrama de velocidade em função da posição de placa na LTQ de Ipatinga. HMD: sensor de posição; HSB: estação de descarepação primária; F3, ...F7, alas dos fornos de reaquecimento; B10: mesa de rolos número 10.

A placa pode ser desenfornada em qualquer uma das alas, denominadas de F3 a F7 na figura 1. A velocidade é constante até a caixa de descarepação, HSB, onde a placa praticamente para, enquanto são enviados dados de setup para o R1. Posteriormente, a velocidade é retomada e, depois, ocorre aceleração até a entrada do R1, quando acontece nova parada antes do primeiro passe. De posse das distâncias relevantes, qualquer gráfico entre as variáveis tempo, velocidade e posição pode ser tratado. Nesse caso, o perfil de velocidade é característico da linha, não dependendo da evolução térmica da placa.

Outro exemplo de representação gráfica do diagrama TPV é mostrado na figura 2, para uma chapa laminada e posteriormente processada no resfriamento acelerado

* Contribuição técnica ao $51^{\circ}$ Seminário de Laminação - Processos e Produtos Laminados e Revestidos, 28 a 31 de outubro de 2014, Foz do Iguaçu, PR, Brasil. 
modificada, equação (9). Reescrevendo-se (9) na forma explícita de diferenças finitas para pontos internos no material obtém-se a equação (10).

$$
\begin{gathered}
c=\frac{\partial \mathrm{H}}{\partial \mathrm{t}} \cdot \frac{\partial \mathrm{t}}{\partial \mathrm{T}} \\
\frac{\partial \mathrm{H}}{\partial \mathrm{t}}=\frac{\lambda_{\mathrm{d}}}{\rho}\left(\frac{\partial^{2} \phi}{\partial \mathrm{x}^{2}}+\frac{\partial^{2} \phi}{\partial \mathrm{y}^{2}}\right) \\
\mathrm{H}_{\mathrm{i}, \mathrm{j}}^{1}=\mathrm{H}_{\mathrm{i}, \mathrm{j}}^{0}+\frac{\lambda_{\mathrm{d}} \Delta \mathrm{t}}{\rho}\left[\frac{2}{\Delta \mathrm{x}_{1}+\Delta \mathrm{x}_{2}}\left(\frac{\phi_{\mathrm{i}-1, \mathrm{j}}-\phi_{\mathrm{i}, \mathrm{j}}}{\Delta \mathrm{x}_{1}}+\frac{\phi_{\mathrm{i}+1, \mathrm{j}}-\phi_{\mathrm{i}, \mathrm{j}}}{\Delta \mathrm{x}_{2}}\right)\right. \\
\left.+\frac{2}{\Delta \mathrm{y}_{1}+\Delta \mathrm{y}_{2}}\left(\frac{\phi_{\mathrm{i}, \mathrm{j}-1}-\phi_{\mathrm{i}, \mathrm{j}}}{\Delta \mathrm{y}_{1}}+\frac{\phi_{\mathrm{i}, \mathrm{j}+1}-\phi_{\mathrm{i}, \mathrm{j}}}{\Delta \mathrm{y}_{2}}\right)\right]
\end{gathered}
$$

$\mathrm{H}_{\mathrm{i}, \mathrm{j}}^{0}, \mathrm{H}_{\mathrm{i}, \mathrm{j}}^{1}$ : entalpia nos instantes de tempo anterior e atual, respectivamente $\left(\mathrm{J} . \mathrm{kg}^{-1}\right)$; $\Delta \mathrm{t}$ : intervalo de solução (s);

$\Delta \mathrm{x}_{1}, \Delta \mathrm{x}_{2}, \Delta \mathrm{y}_{1}, \Delta \mathrm{y}_{2}$ : espaçamentos nas direções $\mathrm{x}$ e $\mathrm{y}$ dos nós anterior e posterior, respectivamente $(\mathrm{m})$;

$\mathrm{i}$, j: identificadores dos nós.

Como o método é explícito, o intervalo máximo de tempo para a solução da equação (10) precisa ser limitado, conforme equação (11), válida para todo o domínio, inclusive no seu interior, onde o coeficiente de transferência de calor é nulo.

$$
\Delta \mathrm{t} \leq \frac{1}{2 \frac{\lambda_{\mathrm{d}}}{\rho \mathrm{c}}\left(\frac{1}{\Delta \mathrm{x}_{1} \Delta \mathrm{x}_{2}}+\frac{\mathrm{h}}{\lambda_{\mathrm{d}} \Delta \mathrm{x}_{1}}+\frac{1}{\Delta \mathrm{y}_{1} \Delta \mathrm{y}_{2}}+\frac{\mathrm{h}}{\lambda_{\mathrm{d}} \Delta \mathrm{y}_{1}}\right)}
$$

h: coeficiente global de transferência de calor entre o material e o meio $\left(\mathrm{W} \cdot \mathrm{m}^{-2} \cdot \mathrm{K}^{-1}\right)$.

Em alguns modelos neste trabalho, foi usado o método de solução numérica por volumes finitos, que foi desenvolvido por Patankar [14]. Enquanto que no MDF a equação diferencial é aproximada por diferenças discretas, no MVF é feito um balanço da grandeza em estudo em cada porção do domínio, chamado de volume finito. Os elementos básicos para a formulação da solução numérica pelo MVF são mostrados na figura 3 , para um problema bidimensional. $O$ ponto $P$ representa $O$ comportamento médio do material no elemento de volume correspondente à área hachurada (o elemento tem espessura unitária). Os pontos $\mathrm{W}, \mathrm{N}, \mathrm{E}$ e $\mathrm{S}$ representam os centros dos elementos de volume vizinhos a $P$, e que trocam calor com este último. O fluxo de calor de dentro ou para fora do elemento ocorre pelas interfaces $w, n, e, s$.

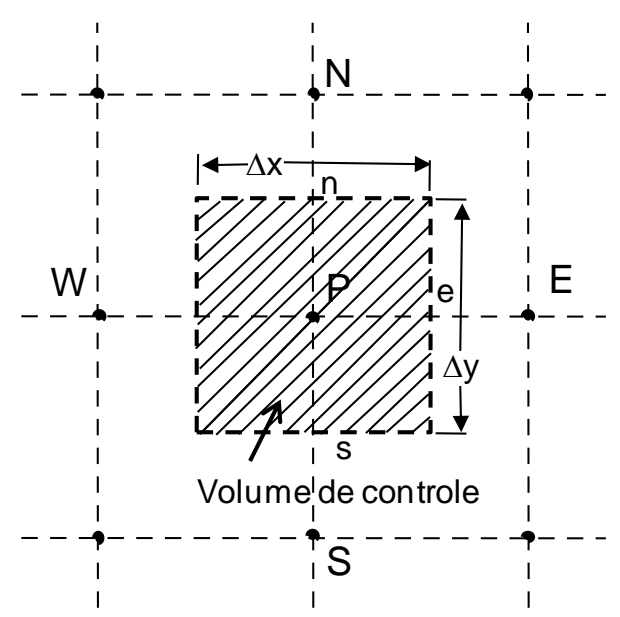

Figura 3. Volume de controle no MVF para um problema bidimensional de condução de calor [14].

* Contribuição técnica ao $51^{\circ}$ Seminário de Laminação - Processos e Produtos Laminados e Revestidos, 28 a 31 de outubro de 2014, Foz do Iguaçu, PR, Brasil. 


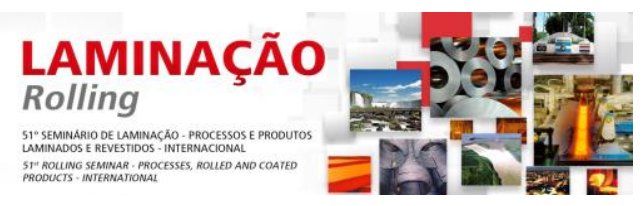

Nesse caso, a forma discretizada reduz-se à equação (12), onde T é a temperatura nos respectivos pontos $\mathrm{P}, \mathrm{W}, \mathrm{N}, \mathrm{E}$ e $\mathrm{S}$, dados como subscritos, e os coeficientes a, relativos a esses pontos, são funções das dimensões do elemento de volume e das propriedades físicas, calor específico, densidade e condutividade térmica do aço. $\mathrm{O}$ termo b é função da geração/absorção de calor no elemento, caso existente, e do estado térmico no instante anterior do ponto $P$. A equação (12) se aplica para 0 interior do domínio. Nas regiões de borda, é necessário introduzir as condições de contorno, alterando-se a forma da equação resultante.

$$
a_{P} T_{P}=a_{E} T_{E}+a_{W} T_{W}+a_{N} T_{N}+a_{S} T_{S}+b
$$

Para cada nó da malha é obtida uma equação semelhante à equação (12). O sistema de equações obtido é então resolvido para cada instante de tempo, havendo para isso uma variedade de alternativas de formulações, por exemplo, formulação implícita, explícita, ou por relaxação. Tem sido adotada a formulação implícita que possui a vantagem de convergir incondicionalmente, apesar de aumento do tempo de solução [14]. A rapidez da solução é maior pela aplicação do algoritmo TDMA (Tridiagonal Matrix Algorithm), com varrimentos alterados de solução nas direções $\mathrm{x}$ e y, o que evita geração de sistemas de equações.

\section{VALIDAÇÃO DOS MODELOS}

Para validação dos modelos foram feitas comparações entre valores de temperatura medidos com os calculados, usando-se significativa quantidade de dados.

Para o caso do processo de reaquecimento, a coleta de dados medidos foi feita através de uma placa instrumentada com termopares em seu interior, aquecida no processo normal. Um coletor de dados, com a devida proteção térmica, fez o registro dos valores medidos. Após o desenfornamento da placa, as curvas de aquecimento medidas foram comparadas com os valores calculados pelo modelo. Na figura 4(a) é mostrado um exemplo de comparação de curvas calculadas e medidas em dois locais no interior da placa, aplicada aos fornos de reaquecimento da Linha de Chapas Grossas (LCG). A curva calculada foi obtida após ajuste dos parâmetros $\varphi_{\mathrm{CG}}^{\mathrm{k}}$, equação (2). As medições feitas pelos termopares no interior da placa instrumentada, durante alguns instantes após seu desenfornamento foram usadas para ajustar os fatores das equações (3) e (5), antes de sua passagem pela estação de descarepação, HSB. Na figura 4(b) estão mostradas comparações de valores previstos pelos modelos com os medidos dessa forma, em local adjacente à superfície. A partir da saída da HSB até o final da etapa de laminação, tanto nas Linhas de Tiras a Quente (LTQ) quanto de LCG, os valores medidos de temperatura foram obtidos dos termopares de controle do processo, que medem a temperatura superficial do material, normalmente em posições na saída da HSB, e na entrada e saída de cada passe de laminação. Isso porque ainda não se conseguiu desenvolver um dispositivo que permita medir as temperaturas internas do esboço ao longo de uma linha de laminação industrial. As medições superficiais estão mais sujeitas a variações e imprecisões devido a interferências tais como, camada de óxido na superfície, presença de água e calibração do pirômetro. Na figura 5, está mostrada uma figura representativa da distribuição de temperatura calculada pelo modelo desenvolvido de evolução térmica da placa, na sua seção transversal, em dois momentos: na saída do forno e na entrada do laminador, para uma condição normal de laminação.

* Contribuição técnica ao $51^{\circ}$ Seminário de Laminação - Processos e Produtos Laminados e Revestidos, 28 a 31 de outubro de 2014, Foz do Iguaçu, PR, Brasil. 


\section{$\underset{\text { Rolling }}{\text { LAMIÇÃO }}$
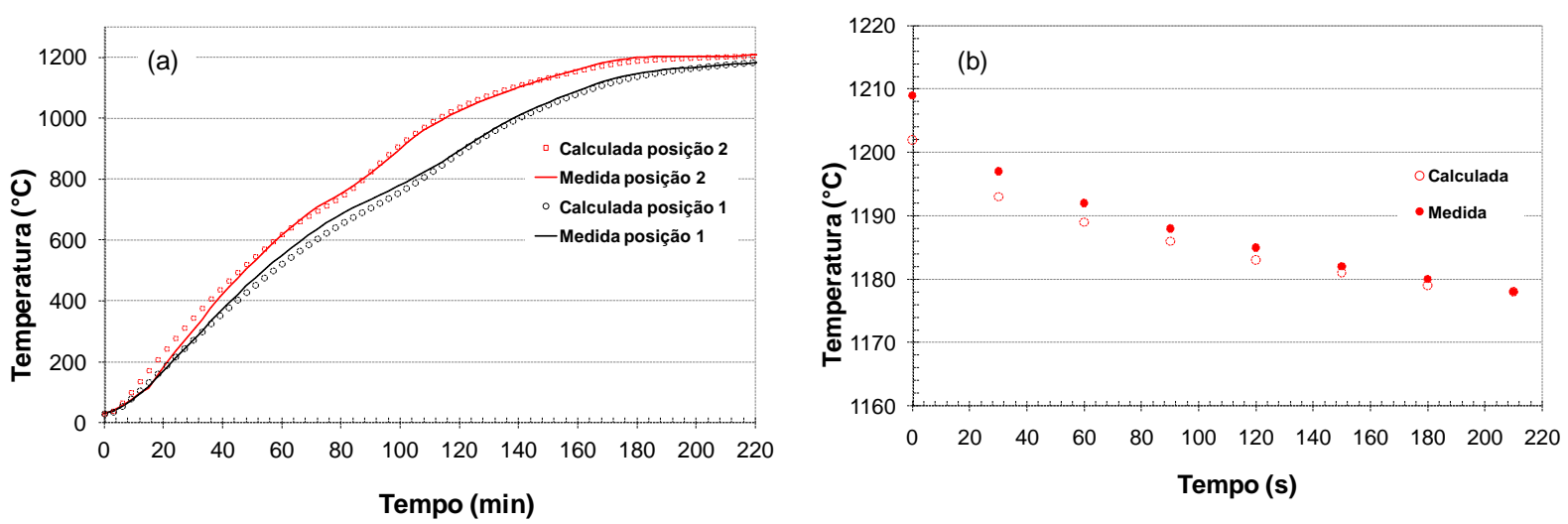

Figura 4. Comparação de curvas medidas e calculadas: (a) processo de reaquecimento de placas na LCG, em duas posições no meio de sua espessura; (b) evolução térmica do desenfornamento à entrada da HSB, na LTQ, em local próximo à superfície da placa.

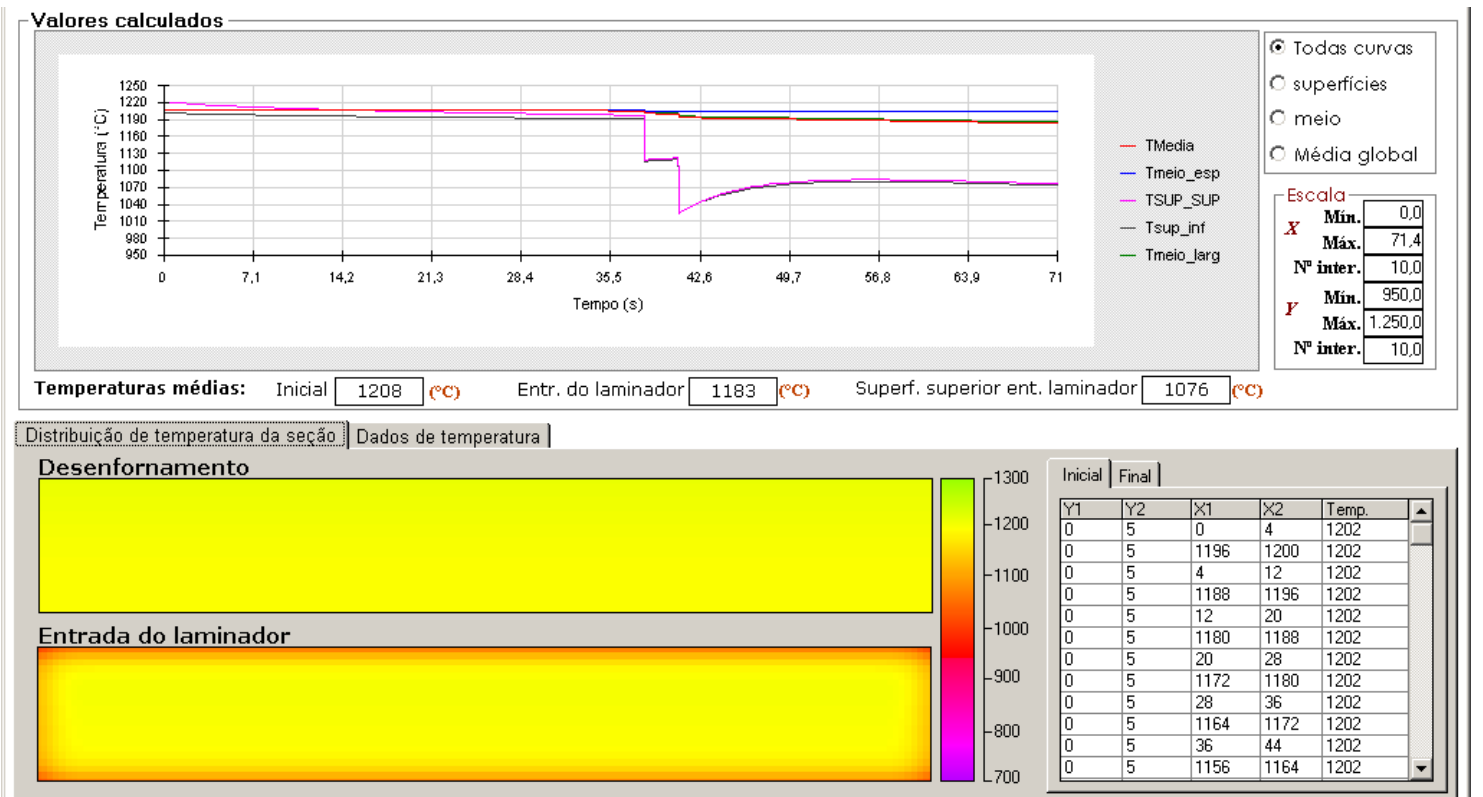

Figura 5. Resultado de simulação da evolução térmica de uma placa na LCG, desde o desenfornamento até a entrada do Laminador. Na parte inferior da figura, está o campo bidimensional (largura $\mathrm{x}$ espessura) de temperatura em dois momentos. Temperaturas: TMedia, média geral na seção; TMeio_esp: média no meio da espessura; TSup_Sup: média na superfície superior, TSup_inf: média na superfície inferior; TMeio_larg, média no meio da largura.

$\mathrm{Na}$ figura 6 são mostradas curvas comparativas medidas e calculadas da temperatura na entrada do passe, para duas sequências de laminação na LCG, obtidas com as formulações utilizadas [15]. A figura 6(a) se refere à laminação convencional de um aço CMn. A figura 6(b) se refere à laminação controlada de um aço microligado. A queda de temperatura durante o tempo de espera entre as etapas de desbaste e acabamento foi corretamente prevista pelo modelo. 


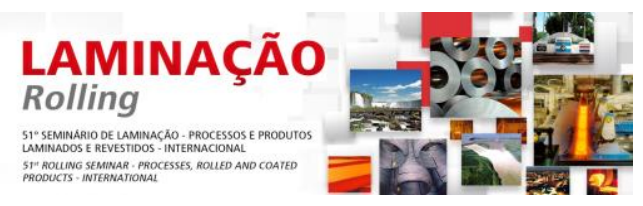

decisão pela interrupção ou não do processamento da placa, uma vez que temperaturas muito baixas podem levar a danos ao equipamento.

Para a etapa de laminação, os modelos desenvolvidos forneceram boa previsão da evolução térmica do laminado. No entanto, sua aplicação foi limitada, pois os resultados foram menos precisos que os fornecidos pelos sistemas de controle online, que possuem várias lógicas de retroalimentação e adaptação durante o processo.

Com relação ao resfriamento do laminado após laminação final de chapas grossas, foram desenvolvidos modelos para o resfriamento acelerado, para o resfriamento final no leito de resfriamento, no caso de chapas não processadas por resfriamento acelerado, e para a evolução térmica durante o empilhamento de chapas, todos na Linha de Chapas Grossas. Especialmente o modelo do resfriamento acelerado tem sido útil para ajustar condições de processo, uma vez que a tecnologia ainda é recente para a Usiminas.

\section{REFERÊNCIAS}

1 Hollander F. A model to calculate the complete temperature distribution in steel during hot rolling. In: Proceedings of the Mathematical Process Models in Iron and Steelmaking Conference, 1973, Amsterdam, 46-79.

2 Santos AA, Schiavo CP, Giacomin CN. Simulação computacional do processo de reaquecimento de placas em fornos de viga móvel. Tecnologia em Metalurgia e Materiais, 2008, 1: 35-39.

3 Panjkivic V, Gloss R. Fast dynamic heat and mass balance model of walking beam reheat furnace with two-dimensional slab temperature profile. Ironmaking and Steelmaking, 2012, 9:190-209.

4 Evans JF, Roebuck ID, Watkins HR. Numerical modeling of hot strip mill runout table cooling. Iron and Steel Engineer, 1993, 1: 50-55.

5 Park IS. Effects of cooling water nozzle array on cooling performance of run out table in hot rolling process. ISIJ, 2013, 1: 71-75.

6 Li HJ, Li ZL, Yuan G, Wang ZD, Wang GD. Development of new generation cooling control systems after rolling in hot rolled strip based on UFC. Journal of Iron and Steel Research International, 2013, 7: 29-34.

7 Devadas C, Samarasekera, IV. Heat transfer during hot rolling of steel strip. Ironmaking and Steelmaking, 1986, 6: 311-321.

8 Santos AA, Giacomin CN. Simulação do processo de resfriamento acelerado de chapas grossas. In: Associação Brasileira de Metalurgia, Materiais e Mineração. Anais do 50ํㅗㄴ Seminário de Laminação, 2013, Ouro Preto. São Paulo: ABM, 2013.

9 The British Iron and Steel Association. Physical constants of some commercial steels at elevated temperatures. London: Butterworths, 1953.

10 Kokado J. On the temperature change of sheet steel during continuous hot rolling. In: Proceedings ICSTIS, Suppl. Trans. ISI, 1971, 11: 750-754.

11 Holman JP. Transferência de Calor. São Paulo, McGraw-Hill do Brasil, 1983.

12 Rohsenow WM. Handbook of heat transfer. New York, McGraw-Hill Book Company, 1973.

13 Thibault J. Comparison of nine three-dimensional numerical methods for the solution of the heat diffusion equation. Numerical Heat Transfer, 1985, 8: 281-298.

14 Patankar SV. Numerical heat transfer and fluid flow. New York, McGraw-Hill, 1980.

15 Santos AA, Giacomin CN. Mathematical simulation of plate rolling at Usiminas: a tool for process enhancement. In: Proceedings 18ª Conferencia de Laminación, 2010, IAS, Rosario/Argentina, 01- 04 novembro. 